Scientific Paper

\title{
Testing of a sag of a dosimetry system rotating with a gantry using the interplay effect between detector motion and MLC motion
}

\author{
Marzena MORAWSKA-KACZYŃSKA ${ }^{1, a}$, Ryszard DĄBROWSKI ${ }^{1}$, Izabela DROZDYK ${ }^{1}$, Paweł KUKOŁOWICZ ${ }^{1}$ \\ ${ }^{I}$ Department of Medical Physics, Maria Sklodowska-Curie Memorial Cancer Center and Institute of Oncology, ul. Wawelska \\ 15, 02-034 Warsaw, Poland \\ ${ }^{a}$ E-mail address: m.morawska@zfm.coi.pl
}

(received 18 May 2017; accepted 31 May 2017)

\begin{abstract}
Purpose: An interplay between detector motion and MLC motion is a source of measurement error, when dose for dynamic arc is measured using a dosimetry system moving relative to the beam central axis during its rotation with a gantry. The purpose of this study is to develop and to evaluate a method of quantitative testing of a sag/flex of such dosimetry systems.

Methods: The method is based on evaluation of relative differences between signals measured for two single arc beams, where a narrow slit field is sliding during gantry movement in opposite directions. The component of a measurement error related to the interplay effect was first assessed based on theoretical computer simulations and then on measurements for four dosimetry systems. The sag pattern of EPID and 2D array was extracted from the measurement results.

Results: The simulations showed a $4 \mathrm{~mm}$ difference in field width and $3.3 \%$ difference in relative signals at beam axis between test beams where the slit field swept over $19 \mathrm{~cm}$ in opposite directions ( sinusoidal sag pattern with amplitude of $1 \mathrm{~mm}$ was assumed). The signal differences exceeding $4 \%$ and $5 \%$ were measured for EPID and 2D array, respectively.

Conclusions: Even relatively small detector sag (less than $1 \mathrm{~mm}$ ) can produce significant measurement error; therefore, the detector sag test should be an obligatory component of a validation of rotating dosimetry systems used for QA of dynamic arcs.
\end{abstract}

Key words: QA; IMAT; dosimetry; interplay.

\section{Introduction}

Volumetric-modulated arc therapy (VMAT) has become a standard modality for delivering highly conformal dose distributions. With the introduction of VMAT, a comprehensive set of relevant (dynamic) performance tests [1-3] has become a part of commissioning and periodic QA tests of accelerators. Due to the high complexity of the VMAT plans, patient specific pre-treatment dosimetry is considered a prerequisite for patient treatment. It has been shown that errors in dose calculations or delivery can be efficiently detected providing that sensitive methods and metrics, as well as robust QA equipment are used [4].

2D dosimetry systems (e.g. EPID or linac head-mounted array detector) have been successfully used for VMAT plan verification [5-7] and for testing the accelerator dynamic performance relevant for VMAT [1,2]. However, like a linac gantry and MLCs [8], these types of dosimetry systems can be affected by the gravity force resulting in a sag. Consequently, the drift of the detector relative to the central beam axis can dynamically change with rotation. As a result of the interplay effect between detector motion and MLC motion, dosimetric errors appear. The importance of the EPID flex in the context of pre-treatment or in-vivo dosimetry of dynamic plans has already been recognised. Some authors $[5,6,9,10]$ have corrected EPID flex while others [11] mechanically stabilised the EPID panel.

In this study, we discuss the dosimetric impact of the interplay effect between detector motion and MLC motion for sliding slit arcs based on the results for two 2D detectors: EPID and head-mounted 2D array detector, and present a novel QA test designed to quantify this effect.

\section{Methods and Materials}

The dosimetric impact of the interplay effect between the detector and the sliding slit field was tested using two methodologies: theoretical computer simulations and experimental measurements. In both cases, a narrow sliding slit field $(1 \mathrm{~cm} \times 20 \mathrm{~cm})$ was moving with constant speed either from the left (LR) or right (RL) side forming a $20 \mathrm{~cm}$ x $20 \mathrm{~cm}$ effective field size. During movement of the sliding slit field, 
the gantry of the linear accelerator was rotating either clockwise (CW) from -90 to 90 degrees or counter clockwise (CCW) from 90 to -90 degrees. The movement of the sliding slit field was aligned with the plane (transverse) and durations of the gantry rotations. The collimator angle was fixed at $0^{\circ}$. The combinations of the sliding slit field movement and gantry rotations created four scenarios abbreviated as: LR-CW, LR$\mathrm{CCW}, \mathrm{RL}-\mathrm{CW}$ and RL-CCW. The necessary treatment fields were created by the iComCAT ${ }^{\mathrm{TM}}$ software (Elekta), a tool designed to create control points for QA beams as required by the linear accelerator. The simulations and measurements were performed for $6 \mathrm{MV}$ photon beams from two Synergy ${ }^{\mathrm{TM}}$ linear accelerators (Elekta), both equipped with 80-leaf MLCi2 collimators and controlled by Integrity v.4. control system. Each test was designed to deliver 200 MUs per scenario. In this study, we defined the detector sag as detector mitigation relative to the central beam axis during gantry rotation.

The methodology for the simulations assumed that the signal of the detector exposed to the rectangular sliding slit photon field is proportional to the time of its exposure to radiation. Therefore, if due to gravitational sag during the gantry rotation, the detector moves relative to the central beam axis with the speed $v_{d}$, its signal may be different for each scenario of the test. Let us assume that the rectangular sliding slit field width is $L$ and the speed of the slit movement across the field is $v_{s}$. The speed of the detector movement, $v_{d}$, will either add to or subtract from the sliding slit field speed, depending on the scenario. For the fixed (still) detector, the time $T$ of being exposed to the photon beam is equal to:

$T=L / v_{s}$

Eq. 1

For scenarios LR-CW and RL-CCW, the exposure time $T 1$ can be described by the following equation:

$T 1=L /\left(v_{s}-v_{d}\right)$

Eq. 2

However, for the scenarios RL-CW and LR-CCW, the exposure time $T 2$ is equal to:

$T 2=L /\left(v_{s}+v_{d}\right)$

Eq. 3

The relative difference of detector signals between the first group and the second group scenarios $\Delta \mathrm{M} / \mathrm{M}$ is described by:

$\Delta M / M=(T 1-T 2) / T$

Eq. 4

Equation 4 can be expressed using sliding slit field and detector speeds, $v_{s}$ and $v_{d}$ as:

$$
\Delta M / M=\frac{2 \cdot v_{s} \cdot v_{d}}{\left(v_{s}-v_{d}\right) \cdot\left(v_{s}+v_{d}\right)}
$$

When the speed of the detector, $v_{d}$, is much smaller than the speed of the sliding slit field, $v_{s}$, Equation 5 can be simplified as:

$$
\Delta M / M=\frac{2 \cdot v_{d}}{v_{s}}
$$

Note that Equation 6 describes the exact solution when the signal for the "still" detector is defined as an average signal measured/simulated for both directions of gantry rotation. For variable speed of the detector movement, $v_{d}$ can be approximated by the average speed of the detector over the time of its effective exposure to the photon beam. When the slit field is sliding over an array of detectors, relative signal difference between two scenarios for each single detector is proportional to the average speed of the array at the time of its exposure. Therefore, relative signal profile differences correlate with a variable speed of the array. Our method of detector sag testing is based on this relation. Relative difference between signal profile measurements for two sliding slit arc fields with opposed field motion directions provides direct information on possible dosimetric error and can be used to estimate the detector sag.

The analysis of the detector sag effect is hampered by potential accelerator-related effects like gantry and MLC gravitational sag. Therefore, relative differences between signal profiles will reflect both interplaying effects: detector sag and MLC sag relative to the beam central axis. To address this issue, a securely head-mounted detector was used to measure the relative signal difference for all previously described test scenarios. However, this time the measured detector signal difference describes only the accelerator-related effects, as the secure attachment prevents any detector movement during gantry rotation, ergo detector speed $v_{d}$ is 0 .

\section{Simulation}

The integrated response of a 1D array to the test beams was calculated using a computer software written in Delphi v.5. for 251 detectors distributed evenly in the crossplane over $250 \mathrm{~mm}$. The test beams were represented by a slit field moving continuously over $19 \mathrm{~cm}$. The slit field intensity was described either by rectangular or Gaussian distribution of 1 $\mathrm{cm}$ width at the $50 \%$ intensity level. The gantry movement was taken into account, by synchronising the slit field movement with the 1D array sag. The sinusoidal sag pattern of the array in the transverse plane with an amplitude of $1 \mathrm{~mm}$ was assumed. For example, the array sag corresponding to LR-CW scenario equalled to $-1,0$ and 1 at the beginning, in the middle and at the end of the narrow beam movement, respectively. The total time of a slit field movement over the array was divided into 10 000 equal time intervals. At each time interval, the following parameters were determined: the position of the slit field centre, the sag-corrected position of each detector and the signal measured by each detector. Simulations were performed for two different responses of detectors: for point detectors and for Gaussian response with a half value width of $5 \mathrm{~mm}$ (close to the response of MatriXX detectors). For a point detector, the calculated signal was equal to a value from the Gaussian slit beam intensity distribution for appropriate coordinate. For Gaussian response, the detector signal was calculated as a convolution of the detector response function and the slit field 
intensity function. The response of the detectors integrated over time was then normalised to the average response of the central detector calculated for two opposite directions of slit field movement.

\section{Measurements}

Measurements of the planar signal distributions for the four test scenarios for two accelerators were performed using the MatriXX ionization chamber array (IBA Dosimetry $\mathrm{GmbH}$ ) attached to the gantry with a dedicated long holder from the same manufacturer and with amorphous silicon electronic portal (EPID) (Elekta Oncology Systems). The measurements were performed at SSDs of $100 \mathrm{~cm}$ and $165 \mathrm{~cm}$, with resolution projected at isocentre of $7.62 \mathrm{~mm}$ and $0.252 \mathrm{~mm}$ for the MatriXX and EPID, respectively. The obtained data was analysed using OmniPro IMRT v1.7 software (IBA Dosimetry $\mathrm{GmbH}$ ). EPID images were transferred from iViewGT software to OmniPro IMRT in a TIFF file format and rescaled using the "Pixel Factor" (coefficient from iViewGT software) in order to obtain dose-proportional data. The maximum sags of the EPID and MatriXX arrays relative to the beam central axis in horizontal gantry position measured with the ruler were both about $1 \mathrm{~mm}$. Additionally, the sag of the EPID was determined by analysing images acquired in cine mode during gantry rotation of a $10 \mathrm{~cm}$ x $10 \mathrm{~cm}$ field with the Elekta Open Air Graticule (Aktina Medical) inserted into the accelerator head. Gantry rotation speed was the same as for the four test scenarios. For successive images, EPID coordinates of the central marker were determined in pixels using iViewGT software, where one pixel corresponded to $0.25 \mathrm{~mm}$. The EPID sag as a function of the gantry angle was calculated as a difference of the marker coordinates between the actual and zero gantry positions.

Measurements of dose distributions for the test scenarios were also performed using Gafchromic EBT3 films. The $5 \mathrm{~cm}$ x $20 \mathrm{~cm}$ pieces of films were attached to the accessory tray at $65 \mathrm{~cm}$ distance from the beam source and covered with $1.2 \mathrm{~cm}$ thick Perspex build-up plate. To reduce the measurement uncertainty, each film was exposed four times which increased the dose delivered to the film to about $1.6 \mathrm{~Gy}$. All films including two calibration pieces were scanned with Epson Perfection V750 PRO scanner using resolution of $0.353 \mathrm{~mm}$. Conversion to dose was performed using an in-house developed program based on three colour channels reading, a noise reduction procedure and corrections of readings in the direction perpendicular to the scanning [12,13]. Finally, the planar doses were imported to the OmniProIMRT software, where a median $5 \times 5$ filter was applied to smooth the data and the coordinates were rescaled from the measured distance of 65 $\mathrm{cm}$ to the isocentre.

Additionally, point dose measurements at the beam central axis were performed for the four scenarios using Farmer (NE2571) ionisation chamber. The chamber was placed in a custom made output calibration phantom mounted at the accessory tray. This phantom is routinely used in our facility for QA measurements of accelerator output at different gantry angles. Results of the measurements with the Farmer chamber and Gafchromic film were considered as reference. They were free of the detector sag effect, as our accessory tray is a very robust mounting platform that did not move relative to the accelerator head during the gantry rotation.

Each set of measurements was performed at least three times and the average results were used for evaluation. For each detector, relative signal profile measurements for two sliding slit arc fields with opposed field motion directions were compared and differences between those signals were calculated. For planar detectors, average relative signals from the region of interest $1.6 \mathrm{~cm} \mathrm{x} 3 \mathrm{~cm}$ around the beam central axis were additionally calculated and compared to the results for point detector.

\section{Detector sag}

Equation 6 describing the relative signal difference of the detector exposed to the sliding slit field moving from left to right and from right to left allows reconstruction of the actual sag pattern of the detector (defined by the transverse shift of the detector relative to beam central axis as a function of the gantry angle). During gantry rotation in $\mathrm{CW}$ direction, the transverse coordinate of the centre of the sliding slit field LR$\mathrm{CW}$ projected at isocentre, $x_{i}$, as a function of gantry angle can be described by the following equation:

$x_{i}=\frac{-g_{i}}{90} \cdot \frac{1000}{S D D} \cdot 95$

For RL-CW scenario, there is a similar equation:

$x_{i}=\frac{+g_{i}}{90} \cdot \frac{1000}{S D D} \cdot 95$

Eq. 8

where $S D D$ is the distance between the photon source and the detector (in $\mathrm{mm}$ ), $g_{i}$ is the gantry angle between -90 and +90 degrees.

Assuming that the measured profiles are symmetrical, the following equation can be derived:

$\Delta s\left(g_{i}\right)=\frac{M 1\left(x_{i}\right)-M 2\left(x_{i}\right)}{M 1\left(x_{i}\right)+M 2\left(x_{i}\right)} \cdot \Delta x$ Eq. 9

where $M 1\left(x_{i}\right)$ and $M 2\left(x_{i}\right)$ are the signals measured by the detector for the sliding field position $\mathrm{x}_{\mathrm{i}}$, for LR-CW (or RL$\mathrm{CCW}$ ) and RL-CW (or LR-CCW) scenarios respectively. $\Delta s\left(g_{i}\right)$ is the transverse shift of the detector at gantry angle $g_{i}$, when sliding slit field travelled a distance of $\Delta x$ during time increment of $\Delta T\left(\Delta s\left(g_{i}\right)=v_{d} \cdot \Delta T\right)$. The total shift of the detector due to sag for an arbitrary gantry angle $g_{N}$ relative to the zero gantry angle can be determined using the following equation:

$s\left(g_{N}\right)=\sum_{i=1}^{N} \Delta s\left(g_{i}\right)=\left(\sum_{i=1}^{N} \frac{M 1\left(x_{i}\right)-M 2\left(x_{i}\right)}{M 1\left(x_{i}\right)+M 2\left(x_{i}\right)}\right) \cdot \Delta x$

Eq. 10 
Equation 10 describes not only the detector sag but it also includes effects of the sag of linear accelerator components (MLC gravitational sag), flex of linac frame, beam profile asymmetry and dose rate variation during gantry rotation. In order to exclude the above effects, the results of the reference system the rigid detector can be utilized (e.g. Gafchromic film). Since the results of the reference systems $\left(M 1_{\text {ref }}\left(x_{i}\right)\right.$ and $\left.M 2_{\text {ref }}\left(x_{i}\right)\right)$ are only affected by the effects related to the linear accelerator, they can be subtracted from the results measured by the non-reference systems like MatriXX array and EPID and the final equation for the tested detector sag is as follows:

$s_{d}\left(g_{N}\right)=\sum_{i=1}^{N}\left(\Delta s\left(g_{i}\right)-\Delta s_{r e f}\left(g_{i}\right)\right)=$

$=\left(\sum_{i=1}^{N}\left(\frac{M 1\left(x_{i}\right)-M 2\left(x_{i}\right)}{M 1\left(x_{i}\right)+M 2\left(x_{i}\right)}-\frac{M 1_{r e f}\left(x_{i}\right)-M 2_{r e f}\left(x_{i}\right)}{M 1_{r e f}\left(x_{i}\right)+M 2_{r e f}\left(x_{i}\right)}\right)\right) \cdot \Delta x$

Eq. 11

Equation 11 was used to calculate the sag for the MatriXX array and EPID detectors. The "reference" sag of the accelerator components measured by the reference system (Gafchromic film) was also calculated using Equation 10.

\section{Results}

\section{Simulation}

Figure 1 presents the results of the calculated relative signal profiles affected by the simulated sinusoidal detector sag pattern. The two curves in Figure 1 represent the calculated relative signal distribution in the transverse plane for LR-CW (RL-CCW) and RL-CW (LR-CCW) scenarios. The presented curves were calculated assuming point response of detectors and Gaussian intensity distribution of a slit field. For rectangular slit field intensity distribution or for detectors with Gaussian response, the simulation results are the same except for differences in a penumbra region. It can be noted that the detector moves at the greatest speed for gantry angle of 0 degree $\left(v_{d} / v_{s}=0.0165\right)$, as can be deducted from the slope of the sag pattern. At that point, the relative dose calculated for the scenario LR-CW is $3.35 \%$ higher than the one for the RL$\mathrm{CW}$ scenario. The results of simulations are consistent with the results obtainable with Equation 6 (3.31\%). Almost the entire calculated profile with the exception of penumbra regions was higher for the scenarios where the sliding slit field moved in the direction of the gantry movement (LR-CW, RL-CCW), as compared to those moving in the opposite directions (RL-CW, LR-CCW). The signal differences in penumbra region represent dose blurring effect. The difference between the calculated profile widths at $50 \%$ of the maximum dose was equal to $4 \mathrm{~mm}$, with width smaller for LR-CW (RL-CCW) profile. The profile width difference was 4 times greater than the sinusoidal sag pattern amplitude and it depended only on the initial and final sag values.

The described dosimetric effect of an interplay between detector motion and MLC motion is proportional to the ratio of the sag-induced detector speed to the sliding slit field speed, hence the sensitivity of the method can be controlled by the latter i.e. a two-fold decrease in the sliding slit field speed would double the relative difference between doses registered for the two scenarios.

\section{Measurements}

Table 1 presents numerical results of the measurements performed with MatriXX array, EPID, Gafchromic film and Farmer chamber around the beam central axis for all four scenarios. The results for all detectors except of Farmer chamber were averaged over the $1.6 \mathrm{~cm} \times 3 \mathrm{~cm}$ region of interest around the beam central axis. The largest differences between the relative signals registered for the four scenarios measured with the MatriXX array and EPID for E1 linear accelerator, were $5.4 \%$ and $4.3 \%$ respectively. The relative signal differences resulting from the "reference" sag of the linear accelerator components are represented by the Gafchromic film and Farmer chamber results. The results indicate that the linear accelerator E2 is more sensitive to those effects than the accelerator E1. The direction of the E2 accelerator-related "reference" sag partly counterbalanced the dose differences caused by the detector sag, whereas the "reference" sag of the E1 accelerator resulted in marginal increases of signal difference.

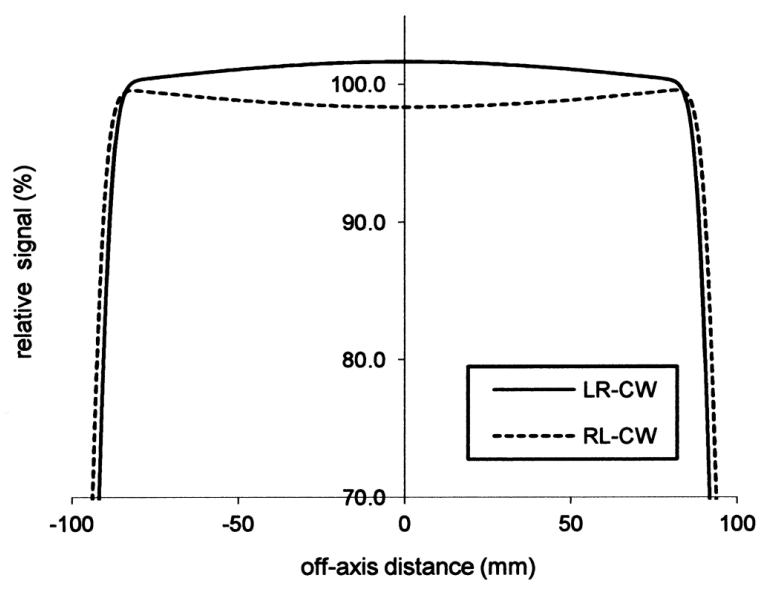

Figure 1. Simulated response of 1D array of point detectors for LR-CW and RL-CW beams for sinusoidal sag pattern of the array 
Table 1. Percentage signals measured at the beam central axis for the test beams. Signals are normalized to the average signal of each detector.

\begin{tabular}{|c|c|c|c|c|c|c|c|c|}
\hline Accelerator & Detector & LR-CW & RL-CW & LR-CCW & RL-CCW & $\Delta_{\max }$ & $\Delta_{\text {mean }}$ & $2 * S D$ \\
\hline \multirow{4}{*}{ E1 } & MatriXX & 102.6 & 97.2 & 97.9 & 102.3 & 5.4 & 4.9 & $0.4 \%$ \\
\hline & iViewGT & 102.0 & 97.9 & 97.9 & 102.2 & 4.3 & 4.2 & $0.7 \%$ \\
\hline & Gafchromic & 100.5 & 99.5 & 100.1 & 99.8 & 1.0 & 0.4 & $0.6 \%$ \\
\hline & Chamber & 100.1 & 99.8 & 99.9 & 100.2 & 0.4 & 0.3 & $0.5 \%$ \\
\hline \multirow{4}{*}{ E2 } & MatriXX & 101.4 & 98.2 & 98.9 & 101.4 & 3.2 & 2.8 & $0.4 \%$ \\
\hline & iViewGT & 99.9 & 99.9 & 100.3 & 99.9 & 0.4 & -0.2 & $0.7 \%$ \\
\hline & Gafchromic & 99.4 & 100.7 & 100.3 & 99.7 & 1.3 & -0.9 & $0.6 \%$ \\
\hline & Chamber & 99.5 & 100.1 & 100.9 & 99.4 & 1.4 & -1.1 & $0.5 \%$ \\
\hline
\end{tabular}

$\Delta$ - difference between results for pairs of test beams (LR-CW, RL-CW) and (LR-CCW, RL-CCW);

$2 * \mathrm{SD}-$ expanded measurement uncertainty $(\mathrm{k}=2)$

(a) film E1

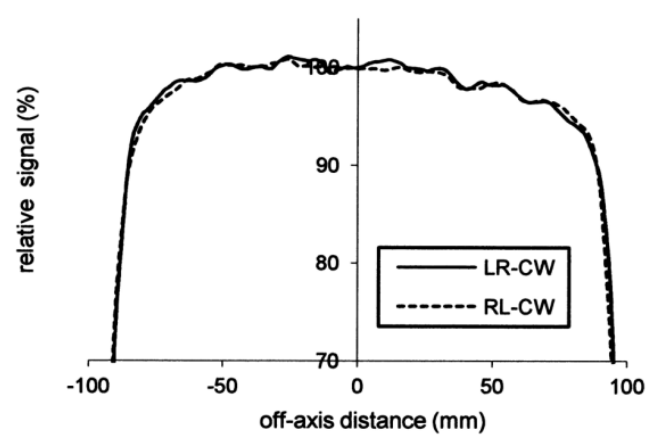

(c) EPID E1

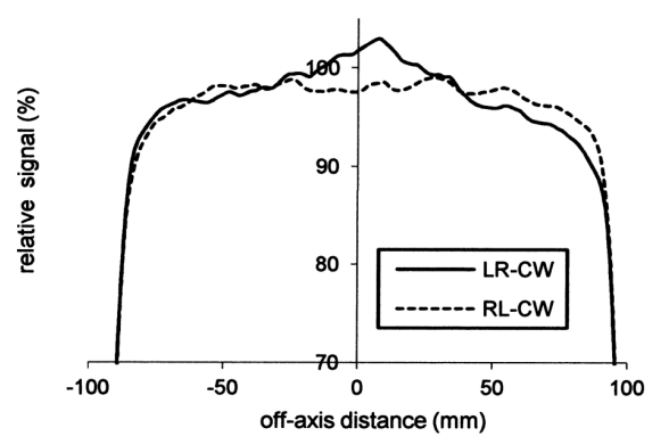

(e) MatriXX E1

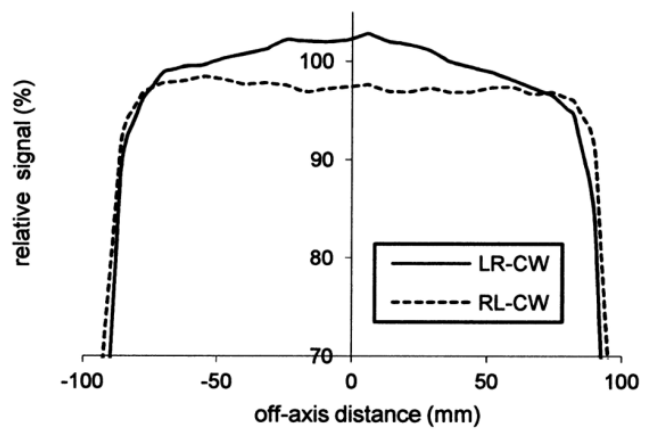

(b) film E2

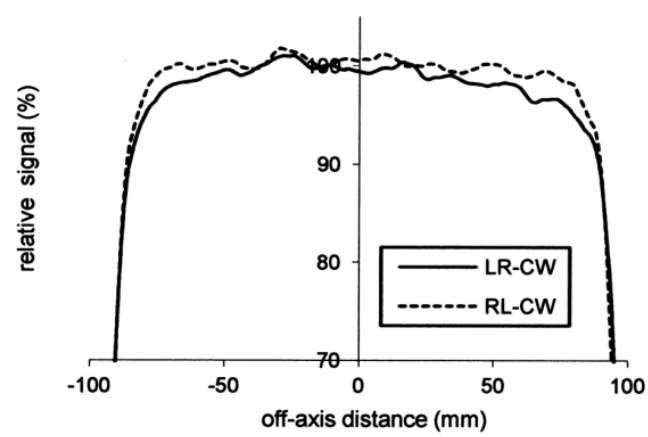

(d) EPID E2

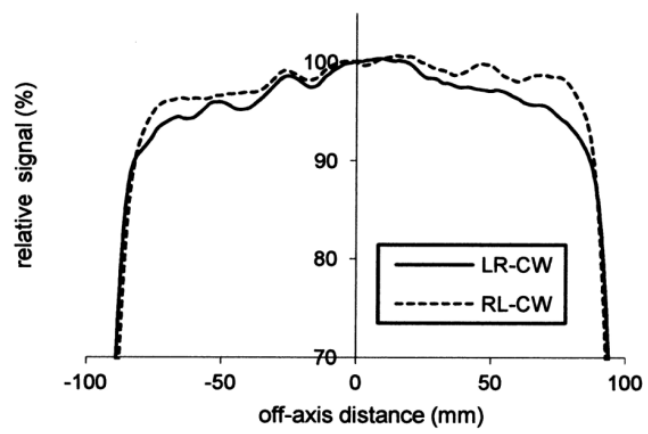

(f) MatriXX E2

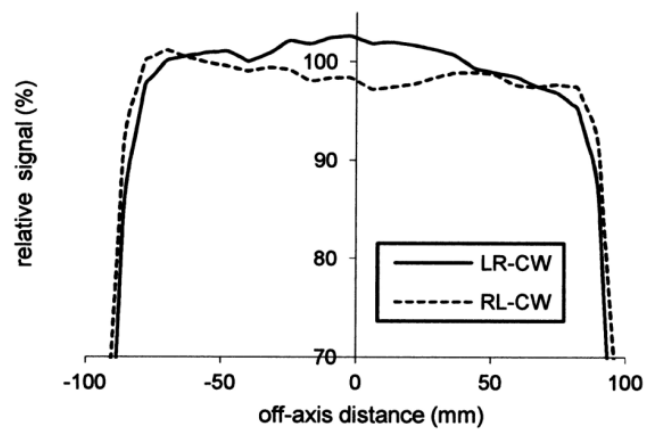

Figure 2. Percentage signal profiles for LR-CW and RL-CW beams measured with Gafchromic EBT3 films (a,b); EPID (c,d) and MatriXX array attached to the gantry with long holder (e,f) for E1 (a, c, e) and E2 (b, d, f) accelerators. 
(a) E1
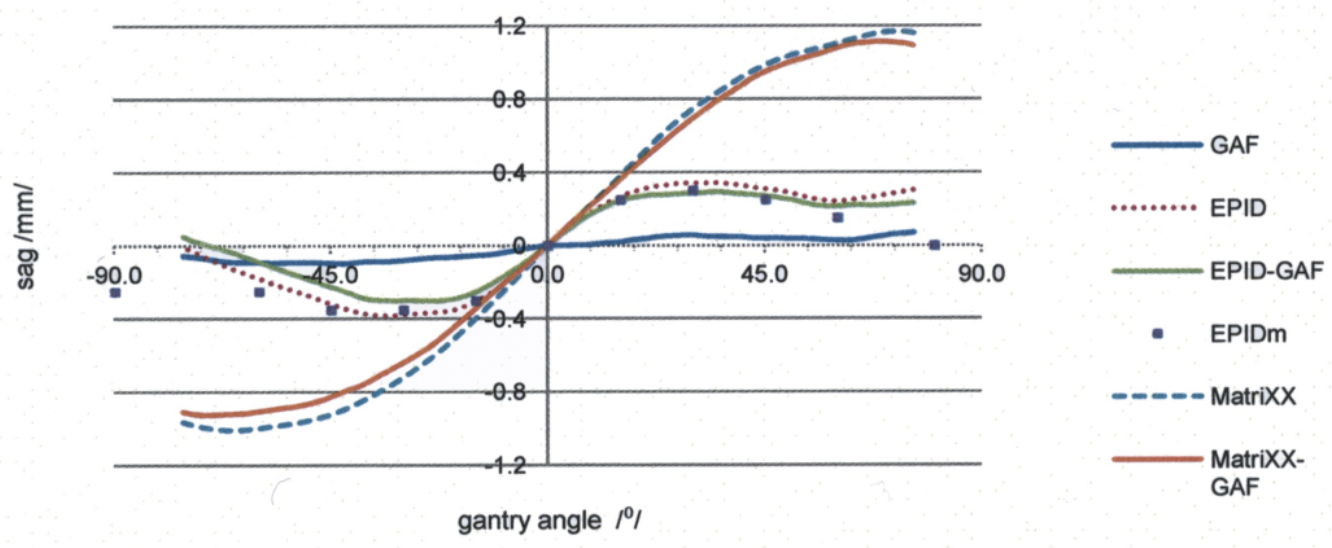

(b) E2

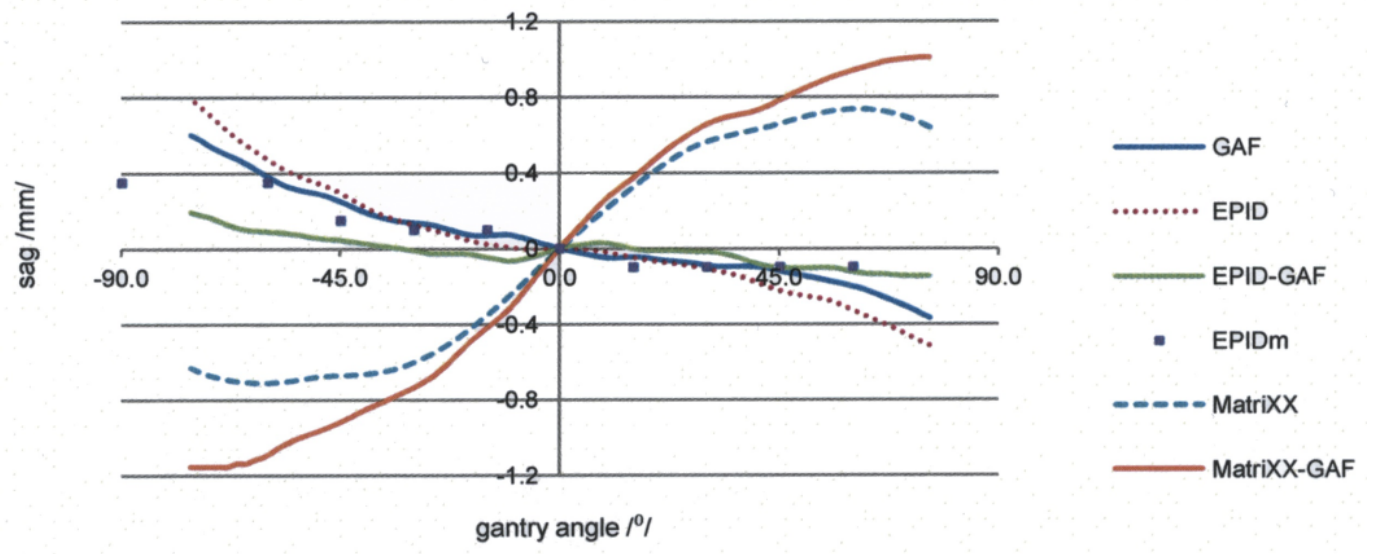

Figure 3. Sag pattern for EPID (EPID-film) and MatriXX array (MatriXX-film) calculated (Eq. 11) based on measurements performed for beams rotating clockwise on two Synergy accelerators: E1 (a) and E2 (b). 'Reference sag' for Gafchromic films (film) and uncorrected sag for EPID (EPID) and for MatriXX array include accelerator-related effects. Independent measurements of the sag for EPIDs (EPIDm) are shown for comparison.

Figure 2 shows the relative signal profiles measured with the MatriXX array, EPID and Gafchromic EBT3 films for two scenarios (LR-CW and RL-CW) for two accelerators, E1 and E2. The relative signal profiles measured for the scenarios LR$\mathrm{CCW}$ and RL-CCW are almost identical and hence not included in Figure 2. Like in the case of the simulation results, the MatriXX array and EPID measurements for the E1 accelerator show the highest relative signal difference at the gantry angle close to 0 degrees. For the E2 accelerator, the MatriXX array measurements also show similar results, however the EPID results appear to be affected differently. The potential explanation is that EPID of the E2 accelerator moves relative to beam central axis against gravity and the measured relative signal difference at the gantry angle close to 0 degrees is counterbalanced by the effects of the "reference" sag. The width of the relative signal profile measured by the EPID on E2 accelerator appears to be marginally larger for LR-CW scenario than for RL-CW one, also suggesting that EPID's sag on this accelerator is negative.

\section{Detector sag}

Figures 3a and 3b show corrected sag patterns for the MatriXX array and EPID, calculated (Equation 11) based on the measurements performed on two linear accelerators, E1 and E2, for gantry rotating clockwise. Uncorrected sag patterns for the MatriXX array and EPID - including the effects of the "reference" sag (Equation 10, only the first part in the parenthesis) as well as the "reference" sag pattern determined from the Gafchromic film measurements - are also shown. Additionally, the EPID panel sag measured for a discrete set of gantry angles with the Open Air Graticule device is shown as the square markers. The "reference" sag was negligible for E1 accelerator but was quite substantial for E2 accelerator. Hence, for E2 accelerator, corrected sag pattern curves for the MatriXX and EPID differ significantly from the uncorrected curves. Although the uncorrected sag pattern curves for the MatriXX array on E1 and E2 accelerators differ significantly, their corrected sag pattern curves are expected to be similar, and they are in agreement within $0.2 \mathrm{~mm}$. The reconstructed 
shape of the corrected sag pattern curve for the EPID on E2 accelerator confirms our earlier hypothesis that the panel movement relative to the beam central axis is performed against the gravitational force during gantry rotation. It is worth mentioning that the corrected sag patterns for the EPIDs determined using Equation 11 agree well with the sag results based on the measurements performed with the Open Air Graticule validating the methodology.

We found that sag amplitude in transverse plane exceeded 1 $\mathrm{mm}$ for our MatriXX array. Sag greater than $1.5 \mathrm{~mm}$ was reported [14] for MapCHECK 2 device also attached to a gantry together with a build-up plate with isocentric mounting fixture. This shows that such heavy dosimetry systems placed in isocentric plane are sensitive to sagging.

\section{Discussion}

Measurements performed with rotating dosimetry systems for an arc beam formed by the rectangular sliding slit field are sensitive to the delivery errors and to the detector variable sagging. Using results of the measurements performed with 2D detectors prone to sagging and with the rigid motionless detectors, we were able to extract the dosimetric distortions caused by the variable sag of $2 \mathrm{D}$ detector. The expected measurement error is proportional to the ratio of the detector speed and MLC speed. For $1 \mathrm{~mm}$ sag the error can exceed 3\%, especially for a low speed of sliding slit field. Our study shows that non-rigid (prone to sagging) rotating dosimetry systems can register erroneous measurement results for the rotating dynamic beams, which may potentially result in misinterpretation of the QA results.

In the pre-treatment QA of VMAT plans, the dosimetric effects of detector sag are less problematic as far as an integrated dose is considered. During delivery of VMAT plans, the irregularly shaped segments formed by MLC leaves usually sweep over the target area in various directions multiple times. For most VMAT plans with such a complex MLC movement, the detector sag-related error would be significantly moderated.
Nevertheless, this moderation may not occur for VMAT beams with low odd number of sweeps where MLC leaves travel slowly. The detector sag becomes apparently a problem for QA of VMAT plans, when time-resolved multiple frames are analysed separately. In such cases, detector sag-related measurement errors for single frames can be comparable to those for sliding slit arcs. Then, the uncorrected sag of rotating dosimetry modalities can significantly deteriorate results and limit the ability to detect potential errors in calculations and delivery.

Detector sag-related errors can be of particular importance for the QA of accelerators, where detectors rotating with the gantry and sliding slit fields might be used to check the accelerator ability to control various beam parameters and their synchronisation in dynamic beams. In such cases, even a small detector sag with amplitude less than $0.5 \mathrm{~mm}$ could increase the occurrence of false negative or false positive types of errors.

\section{Conclusions}

The detector sag test should be an obligatory component of validation of rotating dosimetry systems used for QA of intensity-modulated arcs. The testing method proposed in this study is relatively simple and allows estimation of possible dosimetric error as well as determination of detector sag amplitude and pattern. The proposed method for testing rigidity of rotating dosimetry systems is very sensitive one and can be easily implemented even in small hospitals.

\section{Acknowledgements}

The authors would like to express their gratitude to:

- Romuald Gajewski for thorough revision of the manuscript;

- Staff members of Elekta, Poland, Michal Klos in particular, for their cooperation;

- Grzegorz Cieslik for fruitful discussions.

\section{References}

[1] Ling CC, Zhang P, Archambault Y, et al. Commissioning and quality assurance of RapidArc radiotherapy delivery system. Int J Radiat Oncol Biol Phys. 2008;72(5):575-581.

[2] Kaurin DG, Sweeney LE, Marshall EI, et al. VMAT testing for an Elekta accelerator. J Appl Clin Med Phys. 2012;13(2):55-72.

[3] Netherlands Commission on Radiation Dosimetry (NCS). Code of practice for the quality assurance and control for volumetric modulated arc therapy. Delft, the Netherlands: Netherlands Commission on Radiation Dosimetry, NCS Report 24; 2015.

[4] Nelms BE, Chan MF, Jarry G, et al. Evaluating IMRT and VMAT dose accuracy: Practical examples of failure to detect systematic errors when applying a commonly used metric and action levels. Med Phys. 2013;40(11):111722.

[5] Mans A, Remeijer P, Olaciregui-Ruiz I, , et al. 3D Dosimetric verification of volumetric-modulated arc therapy by portal dosimetry. Radiother Oncol. 2010;94(2):181-187.

[6] Bailey DW, Kumaraswamy L, Bakhtiari M, et al. EPID dosimetry for pretreatment quality assurance with two commercial systems. J Appl Clin Med Phys. 2012;13(4):82-99.

[7] Greer PB. 3D EPID based dosimetry for pre-treatment verification of VMAT - methods and challenges. J Phys: Conf. Ser. 2013;444:012010. 
[8] Du W, Gao S, Wang X, et al. Quantifying the gantry sag on linear accelerators and introducing an MLC-based compensation strategy. Med Phys. 2012;39(4), 2156-2162.

[9] Rowshanfarzad P, Sabet M, O'Connor DJ, et al. Detection and correction for EPID sag during arc delivery using cine EPID imaging. Med Phys. 2012;39(2):623-635.

[10] McCowan PM, Rickey DW, Rowshanfarzad P, et al. An investigation of gantry angle data accuracy for cine-mode EPID images acquired during arc IMRT. J Appl Clin Med Phys. 2014;15(1):187-201.

[11] Iori M, Cagni E, Paiusco M, et al. Dosimetric verification of IMAT delivery with a conventional EPID system and a commercial portal dose image prediction tool. Med Phys. 2010;37(1):377-390.

[12] Micke A, Lewis D, Yu X. Multichannel film dosimetry with nonuniformity correction. Med Phys. 2011;38(5):2523-2534.

[13] Lewis D, Micke A, Yu X, et al. An efficient protocol for radiochromic film dosimetry combining calibration and measurement in a single scan. Med Phys. 2012;39(10):6339-6350.

[14] Jin H, Keeling VP, Johnson DA, et al. Interplay effect of angular dependence and calibration field size of MapCHECK 2 on RapidArc quality assurance. J Appl Clin Med Phys. 2014;15(3),80-92 